See Article page 23.

\section{Commentary: Revival of motor and sensory functions: Is this a catholicon or hollow promise for paraplegia?}

\author{
Devendra K. Agrawal, PhD, MBA, MS, and \\ Vikrant Rai, MBBS, MS, PhD
}

Spinal cord ischemic-reperfusion injury (SIRI) during thoracoabdominal aneurysm repair can lead to changes in motor, sensory, and autonomic functions resulting in neurological deficiency and disability. Minimally invasive surgical procedures limit the ischemic injury, but SIRI remains a distressing complication manifested by paraplegia or paraparesis. ${ }^{1}$ The changing microenvironment after SIRI inhibits axonal regeneration. Bone-marrow-derived mesenchymal stem cells (BM-MSCs), due to their plasticity, can be used as potential therapeutics by modulating the microenvironment. Therapeutic role of MSCs in spinal cord injury is via regulating gliosis, antiapoptosis, inflammation, oxidative stress, angiogenesis, differentiation to neural and glial cells, axonal regeneration, and secretion of growth factors, cytokines, and chemokines. ${ }^{2,3}$ MSCs are administered intracranially/intrathecally or intravascularly with better results for larger lesions via the intravascular route. ${ }^{4}$ Retro-orbital injection of MSCs after ischemic injury plays a protective role in repairing SIRI in rats by preventing autophagy and promoting neurite growth and regeneration. ${ }^{3}$ Preemptive intrathecal injection of MSCs also plays a protective role by stabilizing the blood-spinal cord barrier integrity after SIRI via matrix metallopeptidase 9 and tumor necrosis

\footnotetext{
From the Department of Translational Research, Western University of Health Sciences, Pomona, Calif.

Dr Agrawal is supported by research grants R01 HL144125 and R01HL147662 from the National Institutes of Health. The content of this article is solely the responsibility of the authors and does not necessarily represent the official views of the National Institutes of Health.

Disclosures: The authors reported no conflicts of interest.

The Journal policy requires editors and reviewers to disclose conflicts of interest and to decline handling or reviewing manuscripts for which they may have a conflict of interest. The editors and reviewers of this article have no conflicts of interest.

Received for publication June 24, 2021; revisions received June 24, 2021; accepted for publication June 29, 2021; available ahead of print July 16, 2021

Address for reprints: Devendra K. Agrawal, PhD (Biochem), PhD (Med Sci), MBA, MS (ITM), Department of Translational Research, Western University of Health Sciences, 309 E Second St, Pomona, CA 91766-1854 (E-mail: DAgrawal@ WesternU.edu).

JTCVS Open 2021;7:43-4

2666-2736

Copyright (c) 2021 The Author(s). Published by Elsevier Inc. on behalf of The American Association for Thoracic Surgery. This is an open access article under the CC BY-NC-ND license (http://creativecommons.org/licenses/by-nc-nd/4.0/)

https://doi.org/10.1016/j.xjon.2021.06.022
}

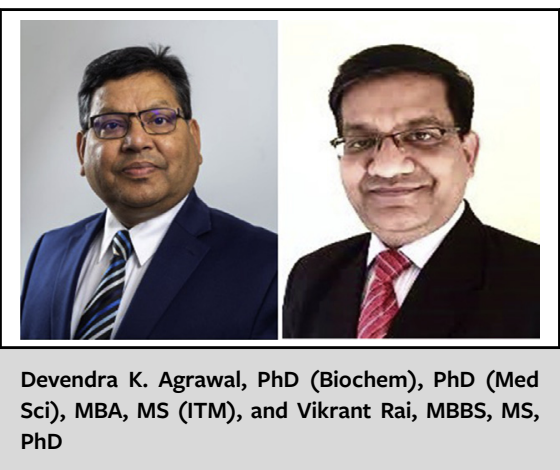

CENTRAL MESSAGE

Preconditioning of mesenchymal stem cells and using mesenchymal-stem-cell-derived extracellular vesicles or exosomes via intravascular route improve recovery in spinal cord ischemiareperfusion injury.

factor- $\alpha$ inhibition. ${ }^{5}$ Increased number of neurons and decreased damage to neurons in animal models and mixed results of improvement in motor activity and sphincter control in some patients, whereas no improvement in others, support the notion of therapeutic use of MSCs; however, warrants an in-depth understanding of the repair mechanisms to enhance therapeutic efficacy, efficiency, reproducibility, and to promote clinical use of MSCs., ${ }^{2,6,7}$

Nakai and colleagues ${ }^{8}$ report improved hindlimb motor function with significantly preserved motor neurons in mice with SIRI and injected intravenously with human BMMSCs by promoting angiogenesis and antiapoptosis and inhibition of proinflammatory cytokines. Using Dil (1,1'-Dioctadecyl-3,3,3',3'-Tetramethylindocarbocyanine Perchlorate)labeled human BM-MSCs revealed localization of MSCs in the ventral horn of the spinal cord; however, the number of surviving MSCs was not evaluated. This is important because apoptosis of transplanted MSCs is a limitation in MSC-based therapy. Preconditioning of MSC with hypoxia effectively increases the survival rate of BM-MSCs via increased HIF- $1 \alpha$ (hypoxia-inducible factor 1-alpha), neurologic function, blood-spinal cord barrier, and tissue damage along with apoptosis inhibition after SIRI. ${ }^{9}$ Administration of simulated microgravity-cultured MSCs improves motor recovery after SIRI in rats. ${ }^{10}$ Recently, the protective effect of MSCs has been attributed to the paracrine effect of MSC-derived extracellular vesicles (EVs)/exosomes, ${ }^{7}$ and cell-free therapy using MSC-exosomes is an exciting novel therapy in spinal injury. 


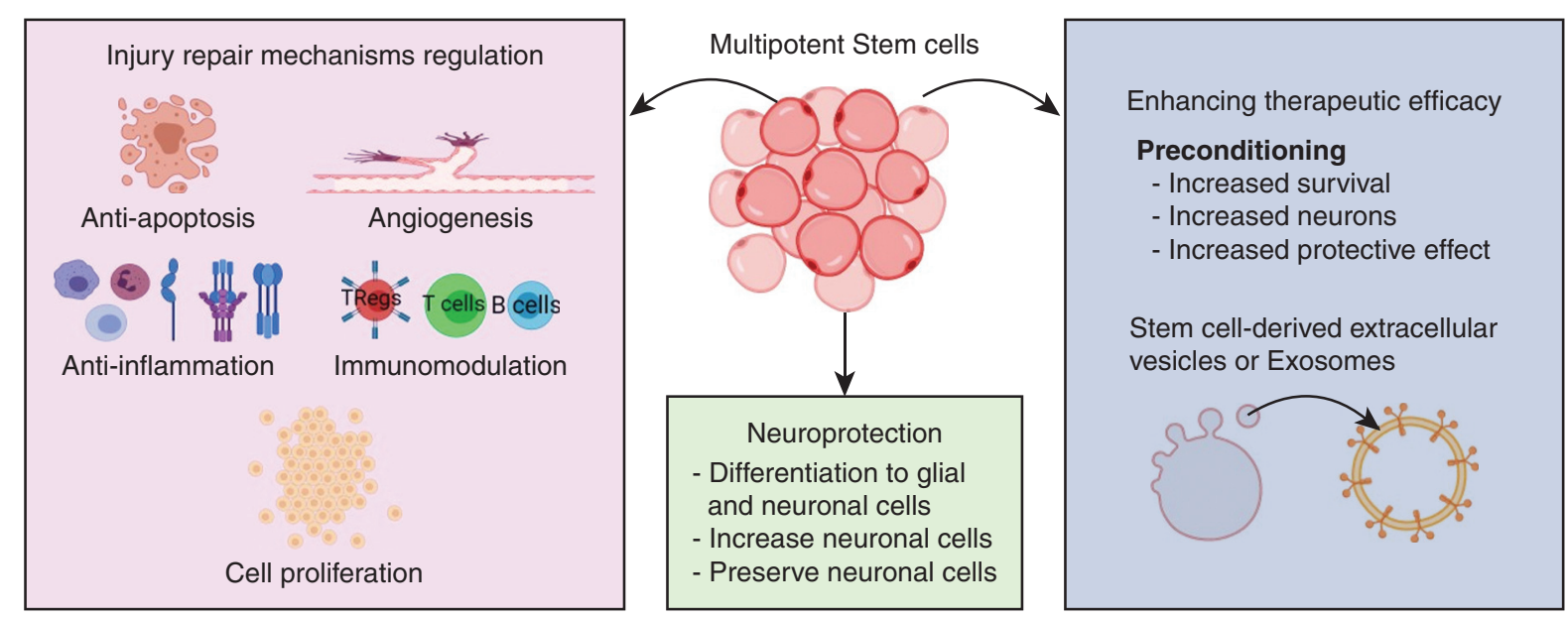

FIGURE 1. Cellular processes involved in the regulation of injury repair mechanisms, neuroprotection, and enhancement of therapeutic efficacy of stem cells.

However, the source for the most potent EVs with therapeutic efficacy needs to be determined. The route of injecting MSCs may have different effects and Nakai and colleagues ${ }^{8}$ have the advantage of using commercially available allogeneic and autologous human BM-MSCs intravenously compared with previous studies ${ }^{11}$ using autologous MSCs administered intrathecally. Investigating the acute and long-term effects of MSC-based therapy on the number of neurons and axonal regeneration is also important. These findings support the feasibility of therapeutic use of MSCs (Figure 1); however, the route of administration, preconditioning of MSCs to enhance survival for longer duration, using MSC-derived $\mathrm{EVs}$, and investigating the long-term effect on neuronal number; generation of action potential; and motor, sensory, and autonomic function; microenvironment of the ischemiareperfusion injury site; host-graft interactions; and the feasibility of therapeutic cell delivery using 3-dimesional scaffolds should be the focus of the follow-up research.

\section{References}

1. Bisdas T, Panuccio G, Sugimoto M, Torsello G, Austermann M. Risk factors for spinal cord ischemia after endovascular repair of thoracoabdominal aortic aneurysms. J Vasc Surg. 2015;61:1408-16.
2. Liau LL, Looi QH, Chia WC, Subramaniam T, Ng MH, Law JX. Treatment of spinal cord injury with mesenchymal stem cells. Cell Biosci. 2020;10:1-17.

3. Yin F, Meng C, Lu R, Li L, Zhang Y, Chen H, et al. Bone marrow mesenchymal stem cells repair spinal cord ischemia/reperfusion injury by promoting axonal growth and anti-autophagy. Neural Regen Res. 2014;9:1665-71.

4. Bliss TM, Andres RH, Steinberg GK. Optimizing the success of cell transplantation therapy for stroke. Neurobiol Dis. 2010;37:275-83.

5. Fang B, Wang H, Sun XJ, Li XQ, Ai CY, Tan WF, et al. Intrathecal transplantation of bone marrow stromal cells attenuates blood-spinal cord barrier disruption induced by spinal cord ischemia-reperfusion injury in rabbits. $J$ Vasc Surg. 2013;58:1043-52.

6. Qu J, Zhang H. Roles of mesenchymal stem cells in spinal cord injury. Stem Cells Int. 2017;2017:5251313.

7. Zhou Y, Wen LL, Li YF, Wu KM, Duan RR, Yao YB, et al. Exosomes derived from bone marrow mesenchymal stem cells protect the injured spinal cord by inhibiting pericyte pyroptosis. Neural Regen Res. 2022;17:194-202.

8. Nakai H, Fujita Y, Masuda S, Komatsu M, Tani A, Okita Y, et al. Intravenous injection of adult human bone marrow mesenchymal stromal cells attenuates spinal cord ischemia/reperfusion injury in a murine aortic arch cross-clamping model. J Thorac Cardiovasc Surg Open. 2021;7:23-40.

9. Luo Z, Wu F, Xue E, Huang L, Yan P, Pan X, et al. Hypoxia preconditioning promotes bone marrow mesenchymal stem cells survival by inducing HIF-1alpha in injured neuronal cells derived exosomes culture system. Cell Death Dis. 2019; 10:134.

10. Kurose T, Takahashi S, Otsuka T, Nakagawa K, Imura T, Sueda T, et al. Simulated microgravity-cultured mesenchymal stem cells improve recovery following spinal cord ischemia in rats. Stem Cell Res. 2019;41:101601.

11. Wang Z, Fang B, Tan Z, Zhang D, Ma H. Hypoxic preconditioning increases the protective effect of bone marrow mesenchymal stem cells on spinal cord ischemia/reperfusion injury. Mol Med Rep. 2016;13:1953-60. 